\title{
Screening for Adverse Childhood Experiences in a Family Medicine Setting: A Feasibility Study
}

\author{
Patricia T. Glowa, MD, Ardis L. Olson, MD, and Deborah J. Johnson, MHA
}

Introduction: The role of adverse childhood experiences (ACEs) in predicting later adverse adult health outcomes is being widely recognized by makers of public policy. ACE questionnaires have the potential to identify in clinical practice unaddressed key social issues that can influence current health risks, morbidity, and early mortality. This study seeks to explore the feasibility of implementing the ACE screening of adults during routine family medicine office visits.

Methods: At 3 rural clinical practices, the 10-question ACE screen was used before visits with 111 consecutive patients of 7 clinicians. Clinician surveys about the use of the results and the effect on the visits were completed immediately after the visits. The presence of any ACE risk and "high-risk" ACE scores $(\geq 4)$ were compared with clinician survey responses.

Results: A risk of ACEs was present in $62 \%$ of patients; $22 \%$ had scores $\geq 4$. Clinicians were more likely to have discussed ACE issues for high-risk patients (score $0-3,36.8 \%$; score $\geq 4,83.3 \% ; P=$. 00). Clinicians also perceived that they gained new information (score $0-3,35.6 \%$; score $\geq 4,83.3 \% ; P=.00$ ). Clinical care changed for a small proportion of high-risk patients, with no change in immediate referrals or plan for follow-up. In 91\% of visits where a risk of ACEs was present, visit length increased by $\leq 5$ minutes.

Conclusions: Incorporation of ACE screening during routine care is feasible and merits further study. ACE screening offers clinicians a more complete picture of important social determinants of health. Primary care-specific interventions that incorporate treatment of early life trauma are needed. (J Am Board Fam Med 2016;29:303-307.)

Keywords: Adult Survivors of Child Adverse Events, Child Abuse, Primary Health Care, Screening, Social Determinants of Health

A large and growing body of literature documents the relationship between adverse childhood experiences (ACEs) and worsened health status and negative life events in adulthood. Initial work by Felitti

This article was externally peer reviewed.

Submitted 15 September 2015; revised 16 February 2016; accepted 19 February 2016.

From the Departments of Community and Family Medicine (PTG, ALO, DJJ) and Pediatrics (PTG, ALO), Dartmouth Geisel School of Medicine, Hanover, NH.

Funding: The Dartmouth Clinical and Translational Science Institute supported the research reported in this feasibility study under award no. UL1TR001086 from the National Center for Advancing Translational Sciences of the National Institutes of Health.

Conflict of interest: none declared.

Disclaimer: The content of this article is solely the responsibility of the author(s) and does not necessarily represent the official views of the National Institutes of Health.

Corresponding author: Patricia T. Glowa, MD, Department of Community and Family Medicine, Dartmouth CO-OP Project, Dartmouth Geisel School of Medicine, HB 7250, 45 Dewey Field Rd, Hanover, NH 03755 (E-mail: Patricia.T.Glowa@hitchcock.org). and colleagues ${ }^{1-3}$ with a patient population from California Kaiser Permanente determined that current health risk behaviors, morbidities, and chronic diseases were predicted by the level of ACE scores. ${ }^{4}$ The 10 ACE questions assess important social determinants of health: childhood abuse, neglect, and household dysfunction. Subsequent studies of other populations have shown that chronic exposure to ACEs, as defined by Felitti et al, affect health outcomes and health care utilization by the time of young adulthood. ${ }^{5-7}$ While ACEs have been widely promoted ${ }^{4,8,9}$ and examined in practice, ${ }^{10}$ community ${ }^{11,12}$ and state-wide surveys, ${ }^{13}$ as well as retrospective chart reviews, ${ }^{13}$ there have, to our knowledge, been no other peer-reviewed publications where ACE questionnaires have been used by clinicians for screening in primary care.

The correlation of increased ACE scores with worsened health status raises the issue of using the ACE questions at primary care visits to identify 
higher-risk subgroups of adult patients and, ultimately, to intervene for improvements in health status and outcomes.

Potential barriers to using ACE questionnaires in the course of office visits include acceptability of the questionnaire to patients and clinicians; whether the clinician learns new, previously unknown information; and potential for the administration of the questionnaire to interfere with the visit logistics, the purpose of the visit, or to increase the length of the visit.

The objective of this preliminary study was to explore the feasibility and utility of using ACE questionnaires as a screening tool in a family practice setting with patients presenting for follow-up of chronic illness or annual physicals. This is a first step in developing care models that address the impact of these early life experiences on adult health.

\section{Methods}

Family medicine practices were recruited to participate in this study at the annual meeting of the Dartmouth CO-OP Primary Care research Network. ${ }^{14}$ The network engages clinicians in Vermont, New Hampshire, and Maine in pragmatic research studies from the planning through implementation stages. A clinician workgroup defined the survey items for a feasibility trial of ACE screening. Seven clinicians in 3 rural primary care practices of the New England Dartmouth CO-OP Primary Care Research Network ${ }^{15}$ subsequently conducted the feasibility study. All clinicians were informed about previous ACE research findings before the study. Two of 3 practices had part-time behavioral health and social work resources available, but they were used predominantly for acute issues. The study was completed during a 2 -week period in 2015, with an additional 2 weeks allowed for part-time clinicians. The goal was to administer the 10-item ACE questionnaire ${ }^{13}$ to a convenience sample of 20 consecutive, non-acutely ill patients per clinician; patients were aged $\geq 18$ years. Data from 1 clinician was omitted because of selective recruitment by this clinician. Recruitment averaged 21 patients per clinician. Visits for acute illness were not screened because of their shorter duration, potentially limiting time for discussion. Nursing staff provided the ACE questionnaires at rooming, and clinicians viewed them during the office visit. Clinicians completed a questionnaire about the office visit immediately after the visit. Clinician and patient questionnaires were linked by study identification numbers. Patient ACE questionnaires collected for analysis had no personal identifiers other than age and sex. The study received approval from the institutional Committee for the Protection of Human Subjects. Study data were collected and managed using REDCap ${ }^{16}$ electronic data capture tools hosted at the Dartmouth-Hitchcock Medical Center.

\section{Data Analysis}

Bivariate statistics were used to compare ACE question responses and risk level for men and women. Felitti and colleagues ${ }^{1,2}$ found a linear relationship with multiple morbidities, but ACE scores $\geq 4$ indicated patients who were at substantially high risk for later morbidity and early mortality. ${ }^{1}$ Thus data were analyzed at 2 levels: (1) any ACE risk present or not and (2) ACE scores with a cut point of $\geq 4$. ACE questionnaires were matched with clinician surveys from the encounter. Clinician responses about the impact of the ACE survey on the visit and changes in care were compared for the 2 levels of ACE scores.

\section{Results}

No patients refused to complete the ACE screening. A total of 127 patients completed the ACE questionnaire. Four patients had incomplete data because they did not have time to finish the screening before the visit, and 12 ACE questionnaires were misplaced and not submitted. Of the subjects, $61 \%$ were women. The average age of patients was $51.9 \pm 17.6$ years (range, 19-87 years). Almost half of visits (48\%) were for chronic illness follow-up, 44\% were for preventive health visits, and $8 \%$ were for other nonacute visits. Data from 111 patients were analyzed.

The responses to each ACE question and the proportion with any risk and high-risk ACE scores are summarized in Table 1 . There were no significant differences by age or sex. Higher-risk ACE scores $(\geq 4)$ were present in $10 \%$ of patients at preventive health visits compared with $30 \%$ at chronic illness follow-up (30\%) and 33\% at other nonacute visits $\left(\chi^{2}=.04\right)$.

Clinician surveys $(n=111)$ reported on interactions during each patient visit. They felt the ACE screener did not interfere with the visit (100\%) and in their opinion it was acceptable to the patient (98\%). Table 2 summarizes the impact of 2 levels of ACE scores, $\geq 1 \mathrm{ACE}$ and $\geq 4$ risks, on the visit. 


\begin{tabular}{cccc}
\multicolumn{3}{c}{ Respondents (\%) } & \\
\hline $\begin{array}{c}\text { Men } \\
(\mathrm{n}=37)\end{array}$ & $\begin{array}{c}\text { Women } \\
(\mathrm{n}=67)\end{array}$ & $\begin{array}{c}\text { Total } \\
\left(\mathrm{n}=111^{*}\right)\end{array}$ & $\begin{array}{c}P \\
\text { Value }^{\dagger}\end{array}$
\end{tabular}

Abuse

While you were growing up, during your first 18 years of life, a parent or other adult in the household often or very often:

Swore at you, insulted you, put you down, or humiliated you, OR acted in a way that made you afraid that you might be physically hurt.

Pushed, grabbed, slapped, or threw something at you, OR ever hit you so hard that you had marks or were injured.

Touched or fondled you or had you touch their body in a sexual way, OR attempted or actually had oral, anal, or vaginal intercourse with you.

Neglect

While you were growing up, during your first 18 years of life, you often or very often felt that:

No one in your family loved you or thought you were important or special,

OR your family didn't look out for each other, feel close to each other, or support each other.

You didn't have enough to eat, had to wear dirty clothes, and had no one to protect you, OR your parents were too drunk or high to take care of you or take you to the doctor if you needed it.

Household dysfunction

While you were growing up, during your first 18 years of life:

Your parents were ever separated or divorced.

$\begin{array}{llll}24.3 & 23.5 & 22.5 & .63 \\ 21.6 & 16.2 & 18 & .50 \\ 8.37 & 25 & 20 & .11\end{array}$

Your mother or stepmother often or very often pushed, grabbed, slapped, or had something thrown at her, OR sometimes, often, or very often kicked, bitten, hit with a fist, or hit with something hard, OR was very repeatedly hit for at least a few minutes or threatened with a gun or knife.

You lived with someone who was a problem drinker or alcoholic or who used street drugs.

A household member was depressed or mentally ill, OR a household member attempted suicide.

A household member went to prison.

18.9

8.1

23.5

20.7

8.1

\begin{tabular}{rrrr}
35.1 & \multicolumn{1}{l}{25} & 27.9 & .30 \\
24.3 & 32.4 & 27.9 & .37 \\
8.1 & 4.4 & 5.4 & .67 \\
59.5 & 64.7 & 62.0 & .51 \\
24.3 & 22.1 & 22.0 & .62 \\
\hline
\end{tabular}

Data were collected during nonacute primary care visits in 2015.

*Seven subjects did not provide a response for gender.

${ }^{\dagger} \chi^{2}$ test.

ACE, Adverse Childhood Experiences.

Clinicians reported the issues detected were discussed, and the ACE questions provided them with new information. This finding was stronger for higher risk ACE scores but clinicians discussed the issues in over 2 thirds of visits for patients having any ACE risk $(\geq 1)$. Use of the questionnaire did not usually change the care in the visit $(5.8 \%$ for $\geq$ 1 risk, $16.7 \%$ for $\geq 4$ risks) or the plan for follow up visits $(1.4 \%)$ and resulted in no new referrals. Using the ACE lengthened the visit but in $90 \%$ of encounters with any ACE risk it added 5 or fewer minutes. With a high risk ACE score $75 \%$ of visits increased by 5 or fewer minutes and none by more than 15 minutes.

\section{Discussion}

Elevated ACE scores were common in these clinical settings: $62 \%$ of patients had $\geq 1 \mathrm{ACE}$ risk and $22 \%$ had a high-risk ACE score of $\geq 4$. The occurrence of any ACE risk is similar to the that among the panel from Kaiser Permanente, of whom 12.5\% had highrisk scores at health appraisals. ${ }^{9}$ In primary care screening these higher scores can be expected with the inclusion of visits for chronic condition manage- 
Table 2. Clinician Responses on the After-Visit Survey $(n=111)$ Collected After Adverse Childhood Experiences Screening

\begin{tabular}{|c|c|c|c|c|c|c|}
\hline & \multicolumn{6}{|c|}{ ACE Risk Level } \\
\hline & $0(\mathrm{n}=42)$ & $\geq 1(\mathrm{n}=69)$ & $P$ Value & $0-3(\mathrm{n}=87)$ & $\geq 4(\mathrm{n}=24)$ & $P$ Value* \\
\hline \multicolumn{7}{|l|}{ This patient's ACE survey results: } \\
\hline Provided new information & $2(4.8)$ & $49(71.0)$ & .00 & $31(35.6)$ & $20(83.3)$ & .00 \\
\hline Changed my clinical care & $0(0)$ & $4(5.8)$ & .11 & $0(0)$ & $4(16.7)$ & .002 \\
\hline Changed plan for follow-up with me & $0(0)$ & $1(1.4)$ & .43 & $1(1.1)$ & $0(0)$ & .60 \\
\hline Changed the referrals made today & $0(0)$ & $0(0)$ & - & $0(0)$ & $0(0)$ & - \\
\hline $\begin{array}{l}\text { Issues detected on the ACE were discussed } \\
\text { during the visit }\end{array}$ & $6(14.3)$ & $47(68.1)$ & .00 & $32(36.8)$ & $20(83.3)$ & .00 \\
\hline \multicolumn{7}{|l|}{ Additional time added to visit (minutes) } \\
\hline $0-3$ & $42(100)$ & $44(63.8)$ & .00 & $77(88.5)$ & $9(37.5)$ & .00 \\
\hline $3-5$ & $0(0)$ & $19(27.5)$ & & $10(11.5)$ & $9(37.5)$ & \\
\hline $5-10$ & $0(0)$ & $4(5.8)$ & & $0(0)$ & $4(16.7)$ & \\
\hline $10-15$ & $0(0)$ & $2(2.9)$ & & $0(0)$ & $2(8.3)$ & \\
\hline$>15$ & $0(0)$ & $0(0)$ & & $0(0)$ & $0(0)$ & \\
\hline
\end{tabular}

Data are $\mathrm{n}(\%)$ unless otherwise indicated.

${ }^{*} \chi^{2}$ test.

ACE, Adverse Childhood Experiences.

ment, since it has been shown that the level of ACE scores are an independent predictor of multimorbidity. ${ }^{17}$ The Kaiser population also included women who were $50 \%$ more likely to have high-risk ACEs, whereas our population showed no sex differences.

ACEs act as chronic life stressors that are not routinely detected in primary care. While it is optimal to address these toxic stressors during childhood, patient screening via a parent questionnaire has not yet been validated. Of relevance to adult ACE screening, higher ACE scores continue to have an impact on later health. Prospective studies of adults show that elevated ACE scores predict hospitalizations for chronic obstructive airway disease 7 years later. ${ }^{18}$ This effect exists after controlling for conventional risk factors (eg, obesity, diabetes, smoking, asthma). In addition to predicting prescription rates for antidepressants 10 years later, ${ }^{19} \mathrm{ACE}$ scores predict adult suicide attempts. After controlling for mental health confounders, each addition point in the ACE score increased the risk of suicide attempts by $50 \%$. There was also an association of ACEs with unintended adult first pregnancies. ${ }^{20} \mathrm{ACE}$ screening has the potential to identify higher-risk populations for targeted health care interventions before these sequelae occur.

While interventions informed by specific trauma in early life have not yet been adapted from mental health settings for use in primary care, this study gives initial information about whether it is feasible to integrate ACE questionnaires into routine office visits for periodic physicals or follow-up of chronic diseases without notably extending the visit. Patients in this exploratory study did not express distress with answering the ACE questions, and clinicians did not report disruptions of the office visit. Administering the questionnaire did not, in itself, extend the office visit. However, the higher the ACE scores, the more likely that the visit was longer. Only $3 \%$ of people with any ACE risk (ACE score $\geq 1$ ) required 10 to 15 minutes of additional visit time. Most visits (91\%) were extended by no more than 5 minutes.

The ACE screening differs from other previsit screening because it provides an expanded personal medical history of the patient, in contrast to other screenings for current tobacco or alcohol use, or an abusive relationship. As such, the history changed the clinical care provided for only 1 of 6 patients with highrisk ACE scores. For family medicine clinicians, specific therapies to address ACEs in this clinical setting are not clearly defined and often are not readily accessible. This may have led to our finding that the plan of care was not altered and referrals were not made.

In this exploratory study we did not collect data on how many patients had already received treatment or how receptive patients are to counseling for traumatic events in early life. While all patients approached completed the survey, we cannot know whether preexisting clinician attitudes about ACEs influenced their responses during the visit. There also are other limitations to be addressed in future studies, including a lack of 
racial diversity, interclinician variability, and a lack of detailed data from patients or subsequent clinic encounters after screening to determine whether screening affects subsequent patient management. This short-term study also did not provide clinicians a way to flag this information as a key aspect of patient history within the electronic health record, which is an important step in using the results of screening during later visits.

This study did, however, indicate that incorporating ACE screening during routine care was feasible and can provide a more complete picture of health determinants that are not usually assessed. It may be useful to link positive ACE scores with screening for other current health risk behaviors associated with high ACE scores (eg, tobacco, alcohol, or other substance abuse; obesity; and depression). Integrating the current understanding of treatment for trauma during early life into the development of clinical care pathways will be important for future progress. With this perspective, interventions to address ACE risks can be developed in primary care settings.

\section{References}

1. Felitti VJ, Anda RF. The relationship of adverse childhood experiences to adult medical disease, psychiatric disorders and sexual behavior: implications for healthcare. In: Lanius R, Vermetten E, eds. The impact of early life trauma on health and disease: the hidden epidemic. New York: Cambridge University Press; 2010:77-87.

2. Felitti VJ, Anda RF, Nordenberg D, et al. Relationship of childhood abuse and household dysfunction to many of the leading causes of death in adults. The Adverse Childhood Experiences (ACE) Study. Am J Prev Med 1998;14:245-58.

3. Dube SR, Felitti VJ, Dong M, Giles WH, Anda RF. The impact of adverse childhood experiences on health problems: evidence from four birth cohorts dating back to 1900. Prev Med 2003;37:268-77.

4. Centers for Disease Control and Prevention. Finding your ACE score. Available from: http://acestudy.org/files/ ACE_Score_Calculator.pdf. Accessed March 18, 2016.

5. Chartier MJ, Walker JR, Naimark B. Separate and cumulative effects of adverse childhood experiences in predicting adult health and health care utlization. Child Abuse Negl 2010;34:454-64.

6. Merksy JP, Topitzes J, Reynolds AJ. Impacts of adverse childhood experiences on health, mental health, and substance use in early childhood: a cohort study of an urban, minority sample in the U.S. Child Abuse Negl 2013;37:917-25.

7. Thompson R, Flaherty EG, English DJ, et al. Trajecto- ries of adverse childhood experiences and self-reported health at age 18. Acad Pediatr 2015;15:503-9.

8. ACEs Connection Network [homepage on the Internet]. Available from: http://www.acesconnection. org. Accessed March 18, 2016.

9. Centers for Disease Control and Prevention. Injury Prevention \& Control: Division of Violence Prevention. 2014. Available from: http://www.cdc. gov/violenceprevention/acestudy/index.html. Accessed March 25, 2016.

10. Chung EK, Nurmohamed L, Mathew L, Elo IT, Coyne JC, Culhane JF. Risky health behaviors among mothers-to-be: the impact of adverse childhood experiences. Acad Pediatr 2010;10:245-51.

11. Cronholm PF, Forke C, Wade R, et al. Adverse childhood experiences: expanding the concept of adversity. Am J Prev Med 2015;49:354-61.

12. Sareen J, Henriksen A, Boulton SL, Afifi TO, Stein MB, Asmundson GJ. Adverse childhood experiences in relation to mood and anxiety disorders in a population-based sample of active military personnel. Psychol Med 2012;43:73-84.

13. Burke NJ, Hellman JL, Scott BG, Weems CF, Carrion VG. The impact of adverse childhood experiences on an urban pediatric population. Child Abuse Negl 2011;35:408-13.

14. Dartmouth Primary Care Research Network [homepage on the Internet]. Available from: http:// www.dartmouthcoopproject.org. Accessed March 18, 2016.

15. Nelson EC, Kirk JW, Bise BW, et al. The Cooperative Information Project: part 1: a sentinel practice network for service and research in primary care. J Fam Pract 1981;13:641/649.

16. Harris PA, Taylor R, Thielke R, Payne J, Gonzalez N, Conde JG. Research electronic data capture (REDCap)-a metadata-driven methodology and workflow process for providing translational resaerch informatics support. J Biomed Inform 2009;42:377-81.

17. Sinnott C, Mc Hugh S, Fitzgerald AP, Bradley CP, Kearney PM. Psychosocial complexity in multimorbidity: the legacy of adverse childhood experiences. Fam Pract 2015;32:269-75.

18. Anda RF, Brown DW, Dube SR, Bremmer JD, Felitti VJ, Giles WH. Adverse childhood experiences and chronic obstructive pulmonary disease in adults. Am J Prev Med 2008;34:396-403.

19. Dube SR, Anda RF, Felitti VJ, Williamson DF, Giles WH. Childhood abuse, household dysfunction and the risk of attempted suicide throughout the life span: findings from adverse childhood experiences. JAMA 2001;286:3089-96.

20. Dietz PM, Spitz AM, Anda RF, Williamson DF, McMahon PM, Santelli JS, Nordenberg DR, Felitti VJ, Kendrick JS. Unintended pregnancy among adult women exposed to abuse or household dysfunction during their childhood. JAMA 1999;282: $1359-64$. 